\title{
Is maternal plasma DNA testing impacting serum-based screening for aneuploidy in the United States?
}

\author{
Glenn E. Palomaki, PhD', Edward R. Ashwood, MD², Robert G. Best, PhD', \\ Geralyn Lambert-Messerlian, $\mathrm{PhD}^{1}$ and George J. Knight, $\mathrm{PhD}^{1}$
}

Purpose: We sought to determine whether tests for fetal aneuploidy based on next-generation sequencing of cell-free DNA in maternal circulation have had an impact on routine serum-based screening in the general pregnant population.

Methods: We compared results from laboratory surveys in 2011 and 2014 that reported types of prenatal serum screening tests and numbers of tests performed. Testing records from two prenatal serum screening laboratories examined temporal trends in the proportion of screened women 35 years of age and older from 2008 (or 2009) to 2014.

Results: The 82 laboratory survey results available for comparison showed that 1.7 million women were screened in 2014 , a $5 \%$ increase over 2011. In the two screening laboratories, the proportion of screened women age 35 and older increased for several years but then experienced reductions of 8 and $18 \%$ by mid- 2014 when compared with the highest rates observed.

Conclusion: As of 2014, maternal plasma DNA testing appears to have had only a minor impact on serum screening rates in the United States. Ongoing surveillance has the potential to determine if, and when, DNA testing begins to replace serum testing as a primary screen for Down syndrome in the United States.

Genet Med advance online publication 2 April 2015

Key Words: cell-free DNA; Down syndrome; next-generation sequencing; screening

\section{INTRODUCTION}

Next-generation sequencing of cell-free DNA in maternal circulation is becoming integrated into routine prenatal practice, with as many as 400,000 tests performed in the United States in 2014. This follows the 2012 recommendation by the American Congress of Obstetricians and Gynecologists (ACOG) to offer DNA testing as a secondary screening option to women already identified as being at high risk for aneuploidy. ${ }^{1}$ However, some commercial laboratories and health-care providers are not restricting testing to high-risk pregnancies. Therefore, it is likely that some portion of the "low-risk" pregnancy population is being provided DNA testing as a primary screening test. In 2013, we published a survey of maternal serum screening laboratories participating in the College of American Pathologists (CAP) external proficiency testing program. ${ }^{2}$ That study documented that $72 \%$ of 4.1 million pregnancies received routine maternal serum prenatal screening services from 131 laboratories. Through multiple methods of ascertainment, we now examine the question of whether the increasing use of DNA testing in prenatal practice has resulted in reduced use of maternal serum-based first- and second-trimester screening in the United States.

\section{MATERIALS AND METHODS}

Three separate data sets were collected and analyzed. One contained nationwide data, another contained data from a large national reference laboratory, and the third contained data from a smaller statewide program in Rhode Island. The nationwide data were derived from the yearly survey of screening laboratories performed as part of the CAP External Proficiency Testing FP Survey (http://www.cap.org). That survey provides five simulated maternal serum challenges three times per year to laboratories offering maternal serum screening for Down syndrome and open neural tube defects. Participants report the specific screening test (e.g., first-trimester combined, secondtrimester quadruple, integrated/sequential) as well as the numbers of each test performed monthly in the first distribution each year. Earlier responses from this survey formed the basis of our recent report on the proportion of women in the United States receiving serum screening services. ${ }^{2}$ Although the identity of individual laboratories was unknown to us, CAP staff were able to link responses from 2001 through 2014 for each participant, allowing for comparisons over time. We matched testing volumes from the 2011 survey to those same participants' 2014 responses. Only laboratories responding to both surveys were included. The change in total tests performed was 
determined, and those results were stratified by total numbers of tests performed.

The next two data sets were derived from prenatal serum screening laboratories with access to individual patient records. Both focused on the assumption that if maternal serum screening were to be impacted, then the likely first indication would be a reduction in the proportion of women age 35 and older being tested. This group has been considered to be of sufficient risk by ACOG to warrant a direct offer of DNA testing without first having serum/ultrasound screening. One of these data sets was compiled by ARUP Laboratories (Salt Lake City, UT). The 50 clients with the largest number of quadruple tests performed in May 2008 were identified and followed through May 2014. The proportion of all quadruple tests performed in women age 35 and older was computed monthly. The second data set was compiled by Women \& Infants Hospital (Providence, RI). The proportion of women age 35 and older who received serum screening (as a percentage) by quarter from 2009 through the second quarter of 2014 was computed. In these latter two data sets, we expected to see increasing rates of uptake by older women from 2008 through 2011 because $\mathrm{ACOG}^{3}$ recommended in 2007 that women of all ages be offered serum/ ultrasound screening. After 2011, those rates were expected to decline because more of these women chose DNA testing as their primary screening test.

\section{RESULTS}

Data from the 2011 CAP survey had already been corrected for certain types of reporting errors and supplemented by results from 2012, as described in a previous publication. ${ }^{4}$ For example, some laboratories reported annual rather than monthly values, or vice versa, and laboratories not reporting in 2011 but reporting in 2012 had the latter results inserted for analysis. In 2011, there were 132 US clinical laboratories with results. In the 2014 survey, 92 laboratories reported results; 80 of them were matched to 2011 results. These 80 participating laboratories were responsible for $88 \%$ of the 1.7 million 2014 tests and for $72 \%$ of all testing reported in 2011. Table 1 (top four rows) shows the changes in number of tests from 2011 to 2014 ((2014 tests - 2011 tests)/2011 tests) for second-trimester, first-trimester, integrated/sequential, and total prenatal testing performed. Overall, second-trimester testing decreased by $3 \%$, first-trimester increased by $126 \%$, integrated/sequential decreased by $10 \%$, and total tests increased slightly by $5 \%$. The majority of laboratories (59\%) found an overall decline in numbers of samples tested.

Table 1 also shows these same data after stratifying by laboratory total test volume using a cutoff of 1,000 samples tested per month (annualized volume of 12,000 tests). Among the 61 smaller laboratories, an overall $12 \%$ average decline in all testing methods was observed, with a small increase of $4 \%$ in the volume of integrated/sequential testing. However, among these same 61 laboratories, 19 (31\%) actually saw an increase over the 3 years. This is in contrast to the larger-volume laboratories $(12,000$ or more tests annually) that reported an overall $8 \%$ increase in testing. All types of testing increased, with the largest percentage increase in first-trimester combined testing (181\%). Most of the decrease in integrated testing and the increase in first-trimester testing were due to one large laboratory with overall numbers that decreased slightly, but it experienced a large shift from integrated/sequential to first-trimester testing.

Results from the large national reference laboratory (ARUP) can be seen in Figure 1, which plots the proportion of women age 35 and older among the screened population. From mid2008 through mid-2013, this proportion steadily increased from 8.6 to $10.0 \%$ (slope $=0.26 \% /$ year increase; $P<0.001$ ).

Table 1 Differences in annual testing volumes between 2014 and 2011 in 80 US laboratories stratified by laboratory testing volume

\begin{tabular}{|c|c|c|c|c|c|}
\hline Total tests performed & $\begin{array}{c}\text { Number of } \\
\text { laboratories }\end{array}$ & $\begin{array}{l}\text { Tests in } \\
2014\end{array}$ & $\begin{array}{c}\text { Tests in } \\
2011\end{array}$ & $\begin{array}{l}\text { Difference from } \\
2011(\%)^{a}\end{array}$ & $\begin{array}{l}\text { Laboratories } \\
\text { increasing (\%) }\end{array}$ \\
\hline First-trimester & 41 & 266,964 & 118,164 & $+148,800(+126 \%)$ & $20(49 \%)$ \\
\hline Integrated/sequential ${ }^{b}$ & 24 & 236,712 & 262,500 & $-25,788(-10 \%)$ & $14(58 \%)$ \\
\hline All & 80 & $1,724,472$ & $1,638,000$ & $+86,472(+5 \%)$ & $33(41 \%)$ \\
\hline Second-trimester & 61 & 166,656 & 185,832 & $-19,176(-10 \%)$ & $18(30 \%)$ \\
\hline Integrated/sequential & 13 & 21,864 & 20,952 & +912 (+4\%) & $5(38 \%)$ \\
\hline All & 61 & 207,288 & 236,664 & $-29,376(-12 \%)$ & $19(31 \%)$ \\
\hline \multicolumn{6}{|l|}{$\geq 1,000 /$ month } \\
\hline First-trimester & 19 & 248,196 & 88,284 & $+159,912(+181 \%)$ & $10(53 \%)$ \\
\hline
\end{tabular}

asecond-trimester is mainly quadruple testing (some alpha-fetoprotein only, triple tests, and quintuple tests). ${ }^{b}$ Integrated/sequential includes serum-integrated, stepwise sequential fully integrated, and contingent models. 'Laboratories testing fewer than 1,000 samples per month. 


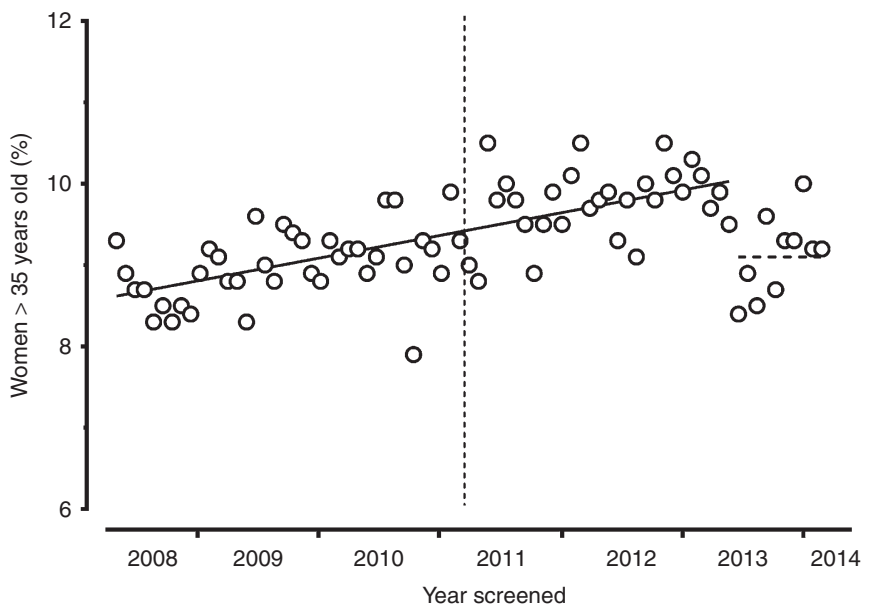

Figure 1 Proportion of a screened population age 35 or older over time from a single, large, national reference laboratory. The $x$ axis shows when screening occurred, with the proportion of women age 35 or older on the $y$ axis. Each dot represents the proportion of the screened women each month that is 35 or older. The solid line indicates the fitted linear regression for the data between mid-2008 (rate of 8.6\%) and mid2013 (rate of 10.0\%). After that time, the data appear to be consistently lower at approximately $9.2 \%$. The dashed vertical line indicates when the recommendation to offer DNA testing to women age 35 and older was promulgated by the American College of Obstetricians and Gynecologists.

However, in the summer of 2013, the rate decreased to approximately $9.2 \%$ and appeared to remain steady thereafter.

At Women \& Infants Hospital, 45,775 women from Rhode Island were screened during the 5.5 years under study (2009 through mid-2014). The average maternal age was 28.7 years. Figure 2 shows the proportion of women age 35 or older in the population. The data fitted a quadratic equation well, with an increasing trend peaking at $19.9 \%$ in 2011 . By mid-2014, the rate was $16.4 \%$, an $18 \%$ decrease.

\section{DISCUSSION}

Next-generation sequencing of maternal plasma for aneuploidy has been offered in the United States by four commercial companies since late 2011 and 2012. Such testing is recommended for use as a secondary screening test in women identified as being at high risk for Down syndrome or other aneuploidy. Several studies have documented the impact on invasive procedures and karyotyping..$^{5-7}$ However, this is the first study aimed at determining whether DNA testing is impacting the high rate of serum screening in the United States, relying on three independent data sets that examine changes in proportions of women receiving serum screening before and after DNA testing was offered or recommended. An ongoing nationwide survey of screening laboratories found that there was an increase in overall serum screening of 5\% between 2011 and 2013. Although the majority of participating laboratories (59\%) saw a decline, larger-volume laboratories saw an increase. This is an ongoing trend that has been documented through previous surveys ${ }^{2}$ and is not likely to be due to the introduction of DNA testing. Individual patient data from two of those participating laboratories showed the

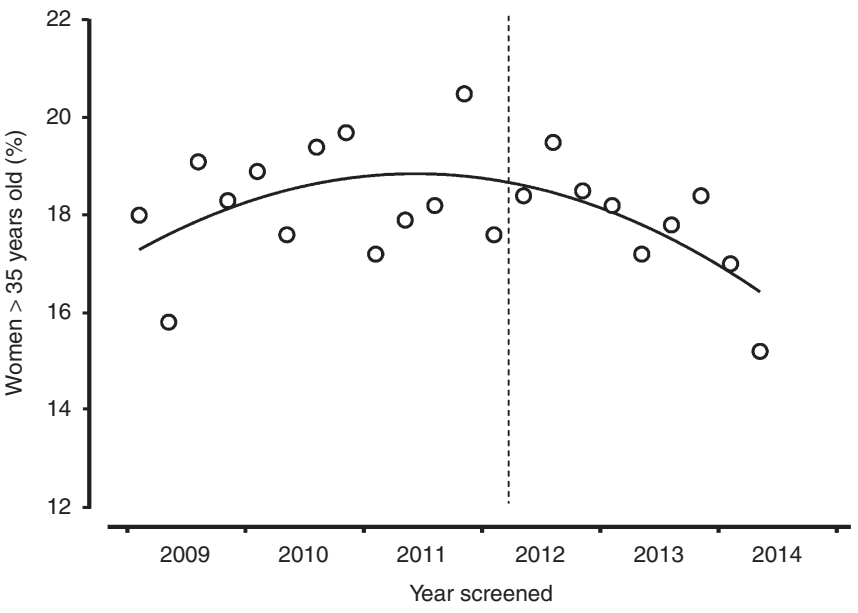

Figure 2 Proportion of a screened population age $\mathbf{3 5}$ or older over time from a single laboratory and stratified by age. The $x$ axis shows when screening occurred, with the proportion of women age 35 or older on the $y$ axis. Each dot represents the proportion of the screened population each quarter that is 35 years of age or older. The solid line indicates the fitted quadratic regression for the data between 2009 and mid-2014 (rate of $10.0 \%$ ). The peak rate was $19.9 \%$, which decreased to $16.4 \%$ by mid2014. The dashed vertical line indicates when the recommendation to offer DNA testing to women age 35 and older was promulgated by the American College of Obstetricians and Gynecologists.

rate of women age 35 and older in the screened population increasing before introduction of DNA testing. However, both show modest decreases after that introduction, and this may be due to the increasing use of maternal plasma DNA testing among women 35 and older. Data from the statewide program in Rhode Island show a peak occurring in 2011, approximately the time DNA testing became available. This is consistent with that program's routine adoption of DNA testing in high-risk pregnancies soon after its introduction in late $2011 .{ }^{8}$

The current analyses have limitations. The nationwide survey data were less complete than those of earlier reports because the current study was restricted to only those laboratories reporting in both 2011 and 2014. Of the 123 respondents who screened 2.7 million pregnancies in the original 2011 report, only 80 participants, who screened 1.6 million women in 2014, were included in the current analysis. It is possible that these data are not representative of all laboratories now offering screening. The proportions shown in Figure 1 from the large reference laboratory are based on screening numbers that have declined $23 \%$ over the 6 years of observation, likely due to attrition of a small number of its largest clients. It is possible that this reduction has some impact on the pattern and may be responsible for the decrease in 2013. Data from the smaller statewide program are based on a consistent and stable population.

Together, these data are consistent with little, if any, reduction in the total numbers of women having serum screening in the United States and with a small but consistent reduction in the proportion of women age 35 and older having serum screening. If several hundred thousand DNA tests are being performed, then who is being tested? Some are women age 35 and older who previously opted for serum screening; others 
would previously have chosen an invasive procedure without any screening, and some may have not had any prenatal testing for aneuploidies. Additionally, some DNA testing is being performed in women younger than age 35 after a positive serum screening test for chromosomal trisomies. Several of these commercial companies will allow low-risk women to be tested, and this may also contribute to the DNA testing numbers, especially among women who would normally accept serum screening even with its relatively high false-positive rate and low positive predictive power. Taken together, these actions could account for the large number of women choosing DNA testing as well as a reduction in women age 35 and older being tested, while still having little or no impact on overall serum screening rates.

What might be expected over the next few years? ACOG and other professional organizations have recommended DNA testing in high-risk pregnancies, but significant barriers may still be restricting higher uptake rates. These could include knowledge about how to obtain DNA testing, questions about the test's performance, and a widespread rejection of reimbursement for this DNA testing application by insurance companies, potentially requiring unacceptably high out-of-pocket costs to be borne by the patient. ${ }^{8}$ Given the typical retail costs of $\$ 800-2,000$, insurance companies may be reluctant to provide coverage until a clear standard of care is established. This may be especially true if there is not a reliable way for insurers to distinguish between testing performed in high-risk pregnancies versus testing in the general population. If high-performance DNA testing is to replace serum screening as the primary screening test in the general population, then the costs of testing will need to decrease. Four studies addressed the costs (not charges, and converted to 2014 US dollars) needed for DNA testing to have a similar cost per case detected currently in place for serum/ultrasound screening. Data from these studies suggest that DNA costs would need to be $\sim 220,{ }^{9} \$ 60,{ }^{10} \$ 600,{ }^{11}$ and $\$ 140-570 .{ }^{12}$ None of these analyses was based on US costs (they were from Belgium, the United Kingdom, the Netherlands, and Australia, respectively) and none directly accounted for the benefit of reduced fetal losses associated with fewer invasive procedures. The lowest cost estimate ${ }^{10}$ is due to the very low cost assigned to firsttrimester combined testing (approximately $\$ 43$ ). All studies focused on Down syndrome. Taking these estimated costs and additional factors into account, reimbursed costs of $\$ 300-400$ might be warranted in the United States. One US-based commercial company now offers a test limited to Down syndrome and trisomy 18 at a significantly reduced price. This strategy might also prove effective in expanding access to lower-risk populations. Distributing the ability to provide such limited testing to academic laboratories may also contribute to both reduced costs and improved insurance coverage. Given the inevitable pressure to offer DNA testing as a primary screening test, research into patient and provider education, predictive values in the general population, handling of test failures/nocalls, and other practical issues surrounding the expansion of services to the broader pregnancy community should become high priorities.

\section{ACKNOWLEDGMENTS}

We thank James E. Haddow, Department of Pathology and Laboratory Medicine, Women \& Infants Hospital (Providence, RI), for helpful comments and critical review. We also thank Pam Provax and other staff at the College of American Pathologists (Northfield, IL) for their help in organizing the survey data.

\section{DISCLOSURE}

G.E.P. was a principal investigator on two independent research projects, both through contracts with Women \& Infants Hospital (Providence, RI). One (NCT00877292), funded by Sequenom (San Diego, CA), was performed from 2009 through 2011. The other (NCT01966991), funded by Natera (San Carlos, CA), was performed from 2012 through 2015 (estimated). G.L.-M. participated in both of these research projects. E.R.A. is Chief Executive Officer of ARUP Laboratories, which has a contract for referral services from Natera. G.E.P., E.R.A., R.G.B., and G.J.K. are members of the College of American Pathologists/American College of Medical Genetics and Genomics Biochemical and Molecular Genetics and Genomics Resource Committee. G.E.P., E.R.A., R.G.B., G.J.K., and G.L.-M. have no stock ownership, patents, or licensing arrangement and have not been paid for publicizing a study. No external funds were provided to support this work.

\section{REFERENCES}

1. ACOG. Committee Opinion No. 545: noninvasive prenatal testing for fetal aneuploidy. Obstet Gynecol 2012;120:1532-1534.

2. Palomaki GE, Knight GJ, Ashwood ER, Best RG, Haddow JE. Screening for down syndrome in the United States: results of surveys in 2011 and 2012. Arch Pathol Lab Med 2013;137:921-926.

3. ACOG. ACOG Practice Bulletin No. 88, December 2007. Invasive prenatal testing for aneuploidy. Obstet Gyneco/ 2007;110:1459-1467.

4. Palomaki GE, Kloza EM, Lambert-Messerlian GM, et al. DNA sequencing of maternal plasma to detect Down syndrome: an international clinical validation study. Genet Med 2011;13:913-920.

5. Chetty S, Garabedian MJ, Norton ME. Uptake of noninvasive prenatal testing (NIPT) in women following positive aneuploidy screening. Prenat Diagn 2013;33:542-546.

6. Taylor JB, Chock VY, Hudgins L. NIPT in a clinical setting: an analysis of uptake in the first months of clinical availability. J Genet Couns 2014;23:72-78.

7. Vahanian SA, Baraa Allaf M, Yeh C, Chavez MR, Kinzler WL, Vintzileos AM. Patient acceptance of non-invasive testing for fetal aneuploidy via cell-free fetal DNA. J Matern Fetal Neonatal Med 2014;27:106-109.

8. O’Brien BM, Kloza EM, Halliday JV, Lambert-Messerlian GM, Palomaki GE. Maternal plasma DNA testing: experience of women counseled at a prenatal diagnosis center. Genet Test Mol Biomarkers 2014;18:665-669.

9. Neyt M, Hulstaert F, Gyselaers W. Introducing the non-invasive prenatal test for trisomy 21 in Belgium: a cost-consequences analysis. BMJ Open 2014; 4 :e005922.

10. Morris S, Karlsen S, Chung N, Hill M, Chitty LS. Model-based analysis of costs and outcomes of non-invasive prenatal testing for Down's syndrome using cell free fetal DNA in the UK National Health Service. PLoS One 2014;9:e93559.

11. Beulen L, Grutters JP, Faas BH, Feenstra I, van Vugt JM, Bekker MN. The consequences of implementing non-invasive prenatal testing in Dutch national health care: a cost-effectiveness analysis. Eur J Obstet Gynecol Reprod Biol 2014;182:53-61.

12. Ayres AC, Whitty JA, Ellwood DA. A cost-effectiveness analysis comparing different strategies to implement noninvasive prenatal testing into a Down syndrome screening program. Aust N Z J Obstet Gynaecol 2014;54:412-417. 